\title{
Comparative expression analysis of the H3K27 demethylases, JMJD3 and UTX, with the H3K27 methylase, EZH2, in Xenopus
}

\author{
AKANE KAWAGUCHI ${ }^{1}$, HARUKI OCHI ${ }^{1,2}$, NORIHIRO SUDOU ${ }^{1,2}$ and HAJIME OGINO ${ }^{1,2, *}$ \\ ${ }^{1}$ Graduate School of Biological Sciences, Nara Institute of Science and Technology (NAIST) and \\ 2JST, CREST, Nara, Japan.
}

\begin{abstract}
The regulated removal of the gene-silencing epigenetic mark, trimethylation of lysine 27 of histone $\mathrm{H} 3$ (H3K27me3), has been shown to be critical for tissue-specific activation of developmental genes; however, the extent of embryonic expression of its demethylases, JMJD3 and UTX, has remained unclear. In this study, we investigated the expression of jmjd3 and utx genes in Xenopus embryos in parallel with that of the H3K27 methylase gene, ezh2. At the blastula stage, jmjd3, utx and ezh2 showed similar expression patterns in the animal cap and marginal zone that give rise to the ectoderm and mesoderm, respectively. The three genes maintained similar expression patterns in the neural plate, preplacodal ectoderm and axial mesoderm during the gastrula and neurula stages. Later, expression was maintained in the developing brain and cranial sensory tissues, such as the eye and ear, of tailbud embryos. These findings suggest that the H3K27 demethylases and methylase may function continuously for progressive switching of genetic programs during neural development, a model involving the simultaneous action of both of the demethylases for the de-repression of silent genes and the methylase for the silencing of active genes.
\end{abstract}

KEY WORDS: H3K27, demethylase, methylase, Xenopus

\section{Introduction}

Epigenetic modifications, such as DNAmethylation, and histone methylation and acetylation, determine chromatin conformation states and modulate the accessibility of DNA target genes to their transcriptional regulators and/or other co-factors (Beisel and Paro, 2011). The trimethylation of lysine 27 of histone $\mathrm{H} 3$ (H3K27me3) is one such epigenetic modification, which is associated with repressed, transcriptionally inactive, chromatin. In mouse embryonic stem (ES) cells, H3K27me3 marks the loci of key developmental regulators, such as Sox and Nkx genes (Bernstein et al., 2006). When the ES cells differentiate into neural stem cells, removal of the H3K27me3 marks from these gene loci precedes their transcriptional up-regulation.

The methylation of H3K27 is catalyzed by Polycomb Repressive Complex 2 (PRC2), a multi-protein complex containing three core subunits: SUZ12, EED and EZH1/2, in vertebrates (Margueron and Reinberg, 2011). The demethylation of H3K27 is catalyzed by two independent enzymes, JMJD3 and UTX, both of which are members of the JUMONJI family proteins that possess the characteristic metalloenzyme-like domain, JmjC (Hubner and Spector, 2011). Previous studies identified crucial roles for the PRC2 methylase complex and the demethylases in various aspects of development. For example, antagonistic functions of PRC2 and UTX regulate the collinear expression of Hox genes (Lan et al., 2007). PRC2 is also necessary for the maintenance of myogenic and epidermal precursor cells, whereas JMJD3 is required for macrophage differentiation (De Santa et al., 2007; Margueron and Reinberg, 2011). These studies suggest that dynamic regulation of the H3K27 methylation state continues throughout development; not only during the early stages as suggested by ES cell studies, but also during the later stages of body axis formation and terminal cell differentiation. However, analysis of the embryonic expression of these epigenetic regulators is very limited, especially in early developmental stages.

Xenopus is an ideal model system for studying epigenetic regulation in vertebrate development, as its large, synchronized,

Abbreviations used in this paper: H3K27, lysine 27 of histone H3; H3K27me3, trimethylation of lysine 27 of histone $\mathrm{H} 3$.

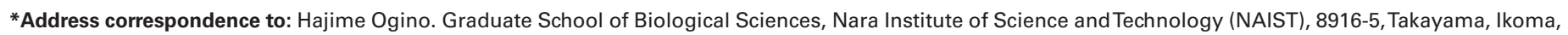
Nara, 630-0192, Japan. Tel: +81-743-72-5552. Fax: +81-743-72-5559. e-mail: ogino@ bs.naist.jp
} 
externally developing embryos facilitate not only gene manipulations but also biochemical analysis of chromatin states at early embryonic stages. While the traditionally used species, Xenopus laevis, is not suitable for genomic analysis due to its large allotetraploid genome, the recent development of its close relative, Xenopus tropicalis, as an alternative model system has largely resolved this issue (Hellsten et al., 2010). In this study, we compare the embryonic expression of jmjd3, utx and ezh2 throughout development in $X$. tropicalis (hereafter referred to as xtjmjd3, xtutx and xtezh2). Expression of ezh2 was previously characterized in $X$. laevis, but histological analysis was limited to the neurula and tailbud stages (Barnett et al., 2001; Aldiri and Vetter, 2009). We found that the H3K27 demethylase and methylase genes, which have antagonistic functions, show quite similar tissue-specific expression patterns in mesoderm and ectoderm lineages.

\section{Results}

\section{Identification of X. tropicalis jmjd3, utx and ezh2 genes}

A search of the $\mathrm{NCBI}$ database resulted in the identification of a $X$. tropicalis cDNA clone (GenBank accession no. BC167994) exhibiting high sequence similarity to the previously identified mouse Jmjd3 (De Santa et al., 2007). This cDNA is largely homologous to an Ensembl $X$. tropicalis gene model, ENSXETT00000034109.

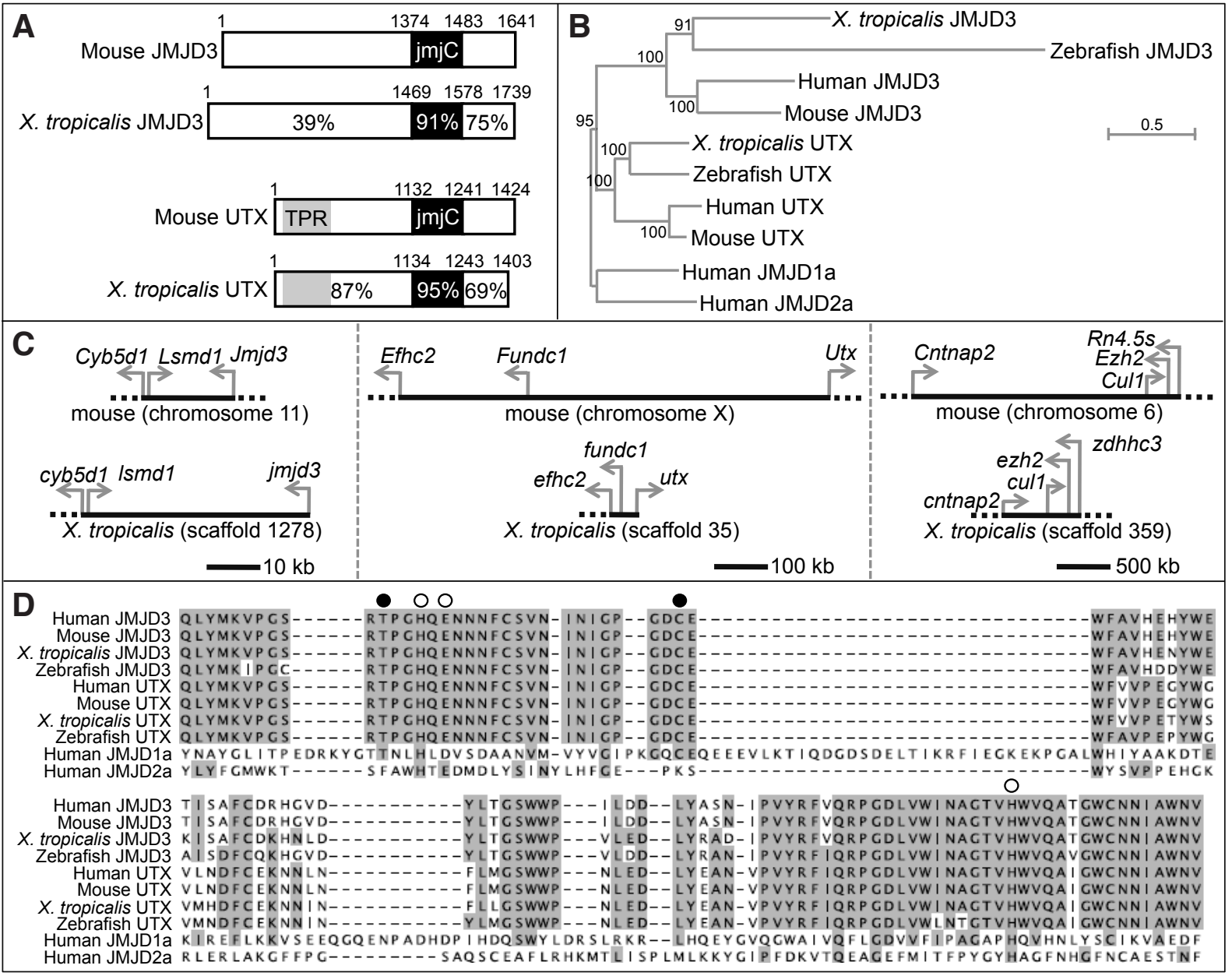

Fig. 1. Identification of $\boldsymbol{X}$. tropicalis JMJD3 and UTX proteins. (A) Schematic comparison between mouse and X. tropicalis JMJD3, and between mouse and X. tropicalis UTX. Numbers in each domain of X. tropicalis JMJD3 and UTX indicate percent identity of the amino acid sequence to the corresponding domain of their mouse orthologs. Amino acid positions are numbered, and JmjC domain and TPR are indicated. (B) The phylogenetic tree analysis of vertebrate JMJD3 and UTX proteins with human JMJD1a and JMJD2a. The tree was constructed using the neighbor-joining method. Scale bar represents distance calculated on the basis of amino acid substitution rates. Numbers on each node indicate percentage bootstrapping values. The GenBank accession numbers of the protein sequences used for this analysis are as follows: zebrafish JMJD3, XP001343575; human JMJD3, NP001073893; mouse JMJD3, NP001017426; zebrafish UTX, XP697746; human UTX, NP066963; mouse UTX, NP033509; human JMJD1a, NP060903; human JMJD2a, NP055478. (C) Synteny analysis of Jmjd3, Utx and Ezh2 in mouse and X. tropicalis. Genes were identified on the X. tropicalis genome assembly (JGI 4.1) and the mouse genome assembly (NCB/ 37) using the UCSC Genome Browser (http://genome.ucsc.edu/). Arrows indicate transcription start sites and the transcriptional orientation of the genes. Only part of the chromosome or scaffold around the Jmjd3, Utx or Ezh2 loci is shown. xtjmjd3 and xtutx locate close to the 3' end of scaffold 1278 and scaffold 35, respectively (left most and middle panels). Although a synteny break resides between the Ezh2 and Rn4.5s or zdhhc3 loci, the synteny from Cntnap2 to Ezh2 is conserved (right most panel). (D) Alignment of JmjC domain sequences of vertebrate JMJD3 and UTX with that of human JMJD1a and JMJD2a. Amino acids that are identical in more than five proteins are highlighted with a gray background. Black and white circles mark the 2-OG-and Fe (II)-binding residues, respectively. These residues are thought to be critical for enzymatic activity of the JUMONJI family proteins (Hubner and Spector, 2011). 
However, open reading frame analysis of the BC167994 led to the identification of a single adenine insertion in the middle of its coding region, which does not exist in the $X$. tropicalis genome sequence and causes a frame shift error at the 1018th amino acid (data not shown). A partial cDNA fragment that contains the middle part of this gene was isolated from $X$. tropicalis tailbud embryos by reverse transcription-polymerase chain reaction (RT-PCR), and sequencing confirmed that the isolated cDNA did not contain the adenine insertion. Therefore we concluded that the inserted adenine in BC167994 was likely a cloning artifact that occurred during the library construction, and thus we reconstructed the full-length coding region (5220 bp) without the adenine using our newly isolated cDNA. We named this reconstructed clone xtjmjd3, and deposited its nucleotide sequence to GenBank (accession no. JN107757). Amino acid sequence comparison between the mouse JMJD3 and the putative XtJMJD3 demonstrates that the catalytic JmjC domain, responsible for demethylation, and the C-terminal domain are both highly conserved ( 91 and $75 \%$ identity, respectively, Fig. $1 \mathrm{~A})$, while the $\mathrm{N}$-terminal domain is largely divergent (39\% identity, Fig. 1A).

We also found $X$. tropicalis cDNA clones in the NCBI database that showed high sequence similarity to the previously identified mouse Utx and Ezh2 (Laible et al., 1997; Greenfield et al., 1998). The clone identified as xtutx (NM001130335) encodes a protein whose $\mathrm{N}$-terminal domain with a tetratricopeptide repeat (TPR), a JmjC domain, and a C-terminal domain share 87, 95 and 69\% amino acid sequence identity, respectively, with their homologous domains in the mouse UTX protein (Fig. 1A). The TPR is thought to mediate protein-protein interactions (Hubner and Spector, 2011). The protein encoded by the clone identified as xtezh2 (NM001017293) shares $94 \%$ amino acid sequence identity with the mouse EZH2 protein and $100 \%$ identity with the previously reported $X$. laevis EZH2 protein (Barnett et al., 2001).

A phylogenetic tree analysis confirmed that XtJMJD3 and XtUTX proteins are more closely related to the previously identified JMJD3 and UTX proteins of other vertebrates, respectively, than to the other members of the JMJD family proteins, such as JMJD1a and JMJD2a (Fig. 1B). Synteny analysis also suggested that $x$ tjmjd3, xtutx and $x$ tezh2 are orthologous of the mouse Jmjd3, Utx and Ezh2 genes, respectively (Fig. 1C). Amino acid sequence alignment shows that the JmjC domains of JMJD3 and UTX are conserved in vertebrates, and are distinct from the JmjC domains of JMJD1a and JMJD2a (Fig. 1D).

\section{Overexpression of $\mathrm{xtjmjd} 3$ and $\mathrm{xtutx}$ reduces $\mathrm{H} 3 \mathrm{~K} 2 \mathrm{27me} 3$ levels in cell culture}

As described above, the amino acid sequences of XtEZH2 and mouse EZH2 are highly conserved, whereas the sequences of XtJMJD3 and XtUTX are somewhat diverged from those of their mouse orthologs. Hence, we examined the demethylase activity of XtJMJD3 and XtUTX by transient transfection of their expression vectors into COS-7 cells followed by fluorescent immunostaining of H3K27me3, as previously performed with mouse JMJD3 (Hong et al., 2007). The transfected cells were distinguished by their expression of a co-introduced GFP vector. When an empty vector was co-transfected with the GFP-expression vector, the level of H3K27me3 staining was indistinguishable between the transfected and untransfected cells (Fig 2 A-C). However, when the cells were transfected with the XtJMJD3-expression vector or the XtUTX-expression vector, significant reduction in $\mathrm{H} 3 \mathrm{~K} 27$ me3 staining was observed (Fig. 2 D-I). A similar observation was made in cells transfected with an expression vector of mouse JMJD3 (Fig. $2 \mathrm{~J}$-L). These results suggest that, as for mouse JMJD3, both XtJMJD3 and XtUTX have H3K27 demethylase activity.

\section{Comparative expression analysis of $x t j m j d 3, x t u t x$ and xtezh2 in embryonic development}

We first examined temporal expression of xtjmjd3, xtutx and xtezh2 by RT-PCR using RNA samples extracted from different developmental stages, ranging from fertilized eggs to tadpoles (Fig. $3 A)$. Transcripts of the three genes were detected at all stages examined, including stages 1-6 preceding the onset of zygotic transcription at the mid-blastula transition (stage 8) (Newport and Kirschner, 1982). This indicates that the xtjmjd3, xtutx and xtezh2 transcripts are maternally supplied, and then maintained by zygotic expression throughout development. Expression levels of xtjmjd3 and $x$ tut $x$ were lowest around the gastrula stage, and then gradually increased by the tailbud stage as the development progressed.

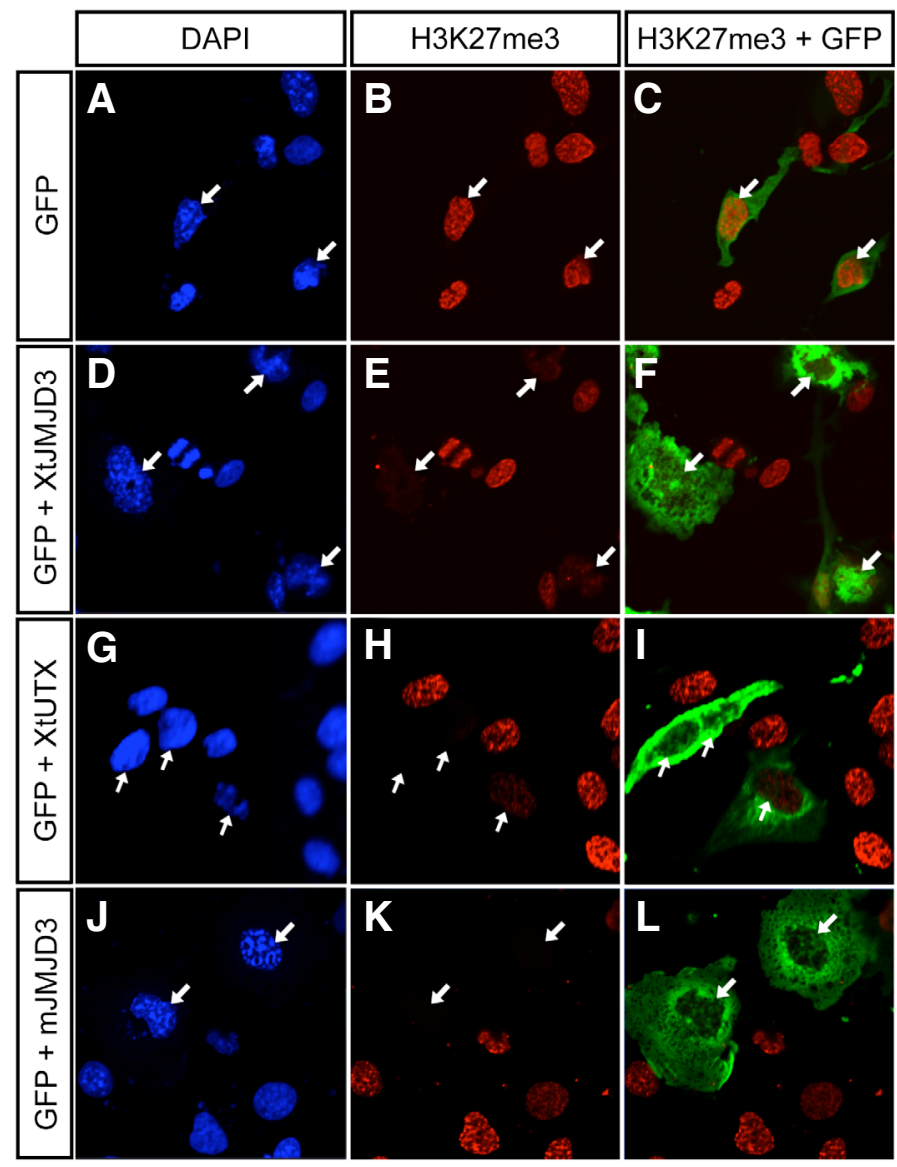

Fig. 2. Overexpression of XtJMJD3 or XtUTX reduced H3K27me3 levels in cell culture. COS-7 cells were transfected with an empty expression vector, pCS2+ (A-C), an XtJMJD3 expression vector, pCS2+xtjmjd3 (DF), an XtUTX expression vector, pCMVSPORT6-xtutx (G-I), or a mouse JMJD3 expression vector, $\mathrm{pCS} 2+\mathrm{mJ}$ mjd3 (J-L). A GFP expression vector, pCAGGS-GFP, was co-transfected in each case to identify the transfected cells. The blue, red and green are nuclear DAPI staining, anti-H3K27me3 antibody staining and anti-GFP antibody staining, respectively. White ar rows indicate nuclei of transfected cells. 
The expression level of xtezh was fairly constant by the gastrula stage, and increased slightly thereafter.

We then analyzed the spatio-temporal expression patterns of xtjmid3, xtutx and xtezh2 by in situ hybridization. At the blastula stage (stage 8), expression of all three genes was detected in the animal hemisphere (Fig. 3 B,G,K). Sections of the embryos showed that both $x t u t x$ and $x t e z h 2$ transcripts mostly localize in the animal cap and marginal zone (Fig. $3 \mathrm{H}, \mathrm{L}$ ), whereas $x$ tjmjd3 transcripts are distributed more broadly through the upper part of the vegetal region (Fig. $3 \mathrm{C}$ ). In the gastrula embryos, the three genes show similar expression patterns in the animal cap and involuting marginal zone (Fig. 3 D,E,E',I,J,J',M,N,N'). This expression domain in the marginal zone appears to correspond to the part of the Spemann organizer (Spemann and Mangold, 1924). After gastrulation, expression of the three genes persists together in the developing neural plate and underlying mesoderm (Fig. 3 O,P,S,T,W,X). Their expression was also evident in the preplacodal ectoderm, which surrounds the anterior margin of the neural plate and later gives rise to the cranial sensory tissues, including the lens, nasal epithelium and inner ear (Schlosser, 2006). High magnification views of the parasagittal section of the anterior region revealed that the anterior endoderm beneath the preplacodal ectoderm also expresses the three genes (Fig. $3 \mathrm{P}^{\prime}, \mathrm{T}^{\prime}, \mathrm{X}^{\prime}$ ). Transverse sections show that the notochord appears to be the center of $x$ tjmjd3 and xtutx expression, and their diffuse expression was detected in the ventral part of the neural plate, proximal part of the paraxial mesoderm and the archenteron roof beneath the notochord (Fig. $3 \mathrm{Q}, \mathrm{U}$ ). In contrast, xtezh2 shows stronger expression in the neural plate than in

Fig. 3. Developmental expression of xtjmjd3, xtutx and xtezh2. (A) Temporal expression was analyzed in whole embryos by RT-PCR at indicated stages. xtodc was used as a loading control (Sindelka et al., 2006). (B-Z) In situ hybridization analysis of blastula, gastrula and neurula embryos. (B, $\mathbf{C}, \mathbf{G}, \mathbf{H}, \mathbf{K}, \mathbf{L})$ Expression at the blastula stage. Lateral views of the whole embryos $(\mathbf{B}, \mathbf{G}, \mathbf{K})$, and their sections are shown $(\mathbf{C}, \mathbf{H}, \mathbf{L})$. The animal pole is at the top. A black triangle in $\mathbf{C}$ indicates dotted expression of xtjmjd3 in the upper part of the vegetal region. (D-E',I-J', M-N') Expression at the gastrula stage. Lateral views of the whole embryos $(\mathbf{D}, \mathbf{I}, \mathbf{M})$, sections $(\mathbf{E}, \mathbf{J}, \mathbf{N})$, and high magnification views of the sections focusing on the involuting marginal zone $\left(\mathbf{E}^{\prime}, \mathbf{J}, \mathbf{N}^{\prime}\right)$ are shown. The animal pole is at the top, and dorsal is to the right. The expression of the $\mathrm{X}$. tropicalis brachyury gene (xtbra) was also examined for comparison with xtjmjd3 (F), since Jmjd3 is involved in the regulation of brachyury in mouse ES cells (see Discussion). (0-0, $\mathbf{S}-\mathbf{U}, \mathbf{W}-\mathbf{Y})$ Expression at the early neurula stage. $(\mathbf{O}, \mathbf{S}, \mathbf{W})$ Frontal views. White broken lines indicate the planes of the parasagittal sections shown in $\mathbf{P}, \mathbf{T}$ and $\mathbf{X}$. ( $\left.\mathbf{P}^{\prime}, \mathbf{T}^{\prime}, \mathbf{X}^{\prime}\right)$ High magnification views of the parasaggital sections in the anterior region. $(\mathbf{Q}, \mathbf{U}, \mathbf{Y})$ Transverse sections of the dorsal part of the embryos. $(\mathbf{R}, \mathbf{V}, \mathbf{Z})$ Expression at the late neurula stage. Lateral views of whole embryos are shown. A white triangle indicates posterior expression of xtezh2. ac, animal cap; mz, marginal zone; dbg, dorsal blastoporal groove; anp, anterior neural plate; ppe, preplacodal ectoderm; np, neural plate; pp, precaudal plate; dm, dorsal mesoderm; ae, anterior endoderm; nc, notochord; pm, paraxial mesoderm; ar, archenteron roof; br, brain; mnc, migrating neural crest. the underlying tissues including the notochord, and the expression patterns are uniform in each tissue (Fig. 3Y).

In the late neurula, expression of the three genes continues in the developing neural tissues, including the eye, brain, and neural crest cells migrating into the branchial arch (Fig. 3 R, V,Z). At the tailbud stage, expression patterns of all three genes appear very similar from the outside (Fig. 4 A,G,M). Transverse sections of the head region of tailbud embryos show that the three genes are expressed in the optic cup, mesencephalon, diencephalon, rhombencephalon, otic vesicle, and migrated neural crest cells, but are no longer expressed in the notochord (Fig. 4 B,D, D, H,J, $\left.J^{\prime}, N, P, P^{\prime}\right)$. In the eye, the three genes show broad expression in the optic cup, except in its outer layer (Fig. 4B', H',N'). In the lens, expression of $x t j m j d 3$ is evident, but expression of other genes is faint. Transverse sections of the trunk region show that $x t j m j d 3$ and
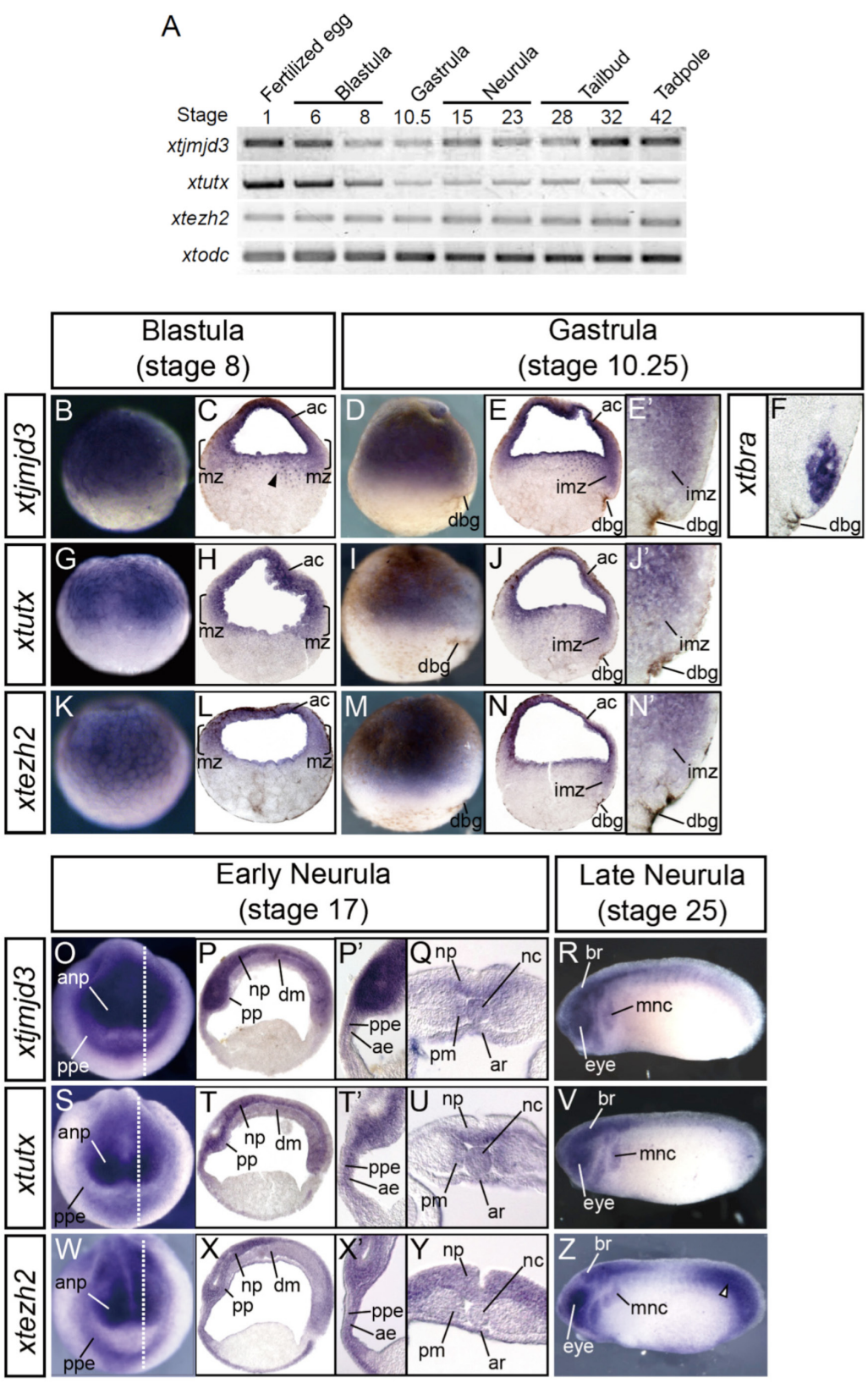


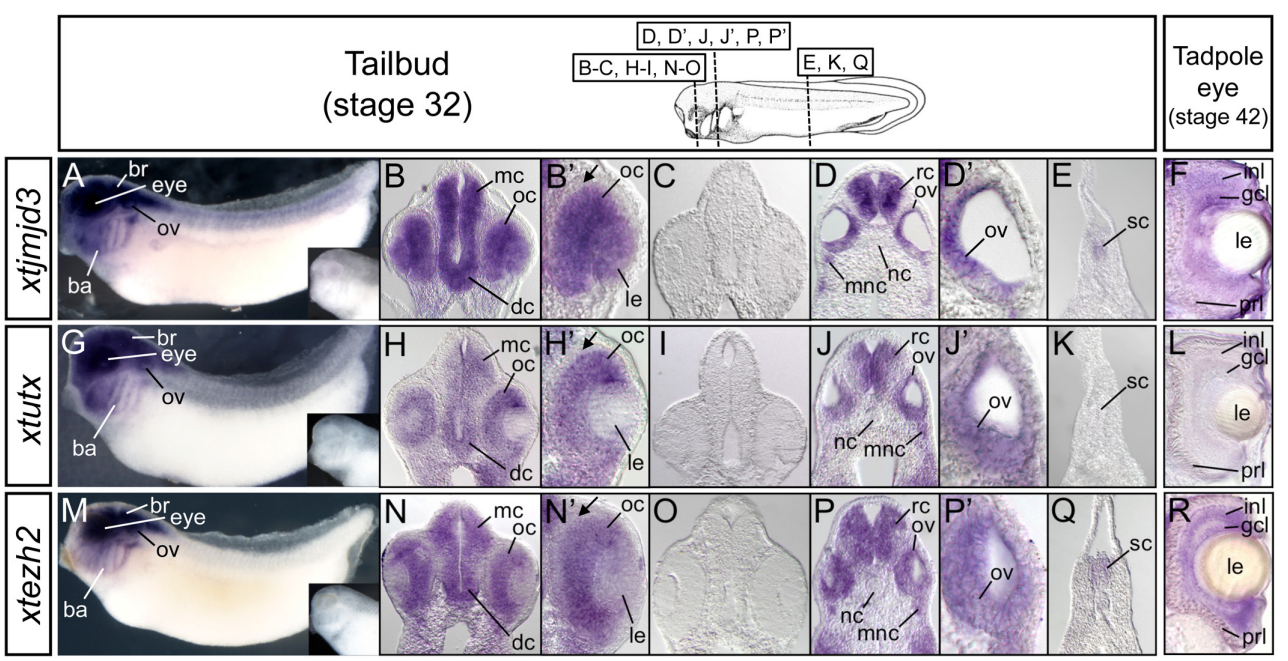

Fig. 4. Expression of $x$ tjmjd3, xtutx and $x t e z h 2$ in tailbud embryos and tadpoles. $(\mathbf{A}, \mathbf{G}, \mathbf{M})$ Lateral views of tailbud embryos subjected to whole-mount in situ hybridization. Insets show embryos hybridized with a sense probe for $\mathrm{xtjmjd} 3$, xtutx or xtezh2. (B-E,H-K,N-Q) Transverse sections subjected to in situ hybridization. The planes of the sections are illustrated in the top white box. (B', $\left.\mathbf{H}^{\prime}, \mathbf{N}^{\prime}\right)$ High magnification views of eyes in the transverse sections shown in $\mathbf{B}, \mathbf{H}$, and $\mathbf{N}$. Arrows indicate the outer layer of the optic cup. $(\mathbf{C}, \mathbf{l}, \mathbf{O})$ Transverse sections hybridized with a sense probe for xtjmjd3, xtutx or xtezh2. (D',J','P') High magnification views of otic placodes in the transverse sections shown in $\mathbf{D}, \mathbf{J}$ and $\mathbf{P}$. $(\mathbf{F}, \mathbf{L}, \mathbf{R})$ Transverse sections of tadpole eyes subjected to in situ hybridization. br, brain; ov, otic vesicle; ba, branchial arch; mc, mesencephalon; dc, diencephalon; oc, optic cup; le, lens; mnc, migrating neural crest; rc, rhombencephalon; nc, notochord; sc, spinal cord; gcl, ganglion cell layer; inl, inner nuclear layer; prl, photoreceptor layer.

xtezh2, but not xtutx, are expressed in the spinal cord (Fig. $4 \mathrm{E}, \mathrm{K}, \mathrm{Q}$ ).

We also examined the expression of xtjmjd3, xtutx and xtezh2 in tadpole eyes in which differentiated retinal layers were established. At this stage, xtezh2 expression was distinct in the ganglion cell layer and inner nuclear layer, but appeared to be absent from the photoreceptor layer (Fig. 4R). Low expression of xtjmjd3 was detected in the ganglion cell layer and inner nuclear layer (Fig. $4 \mathrm{~F})$, but xtutx expression was not detected in any of the retinal layers (Fig. 4L).

\section{Discussion}

Our expression analysis revealed that the transcripts of xtjmid3, $x$ tutx and $x t e z h 2$ preferentially localize to the animal hemisphere by the early gastrula stage. It is not known whether the transcripts are translated into proteins at these stages. However, this localized expression of the H3K27 methylase, xtezh2, may explain the previous findings that genes preferentially expressed in the vegetal hemisphere, such as vegtand sox $17 \alpha$, are marked with $\mathrm{H} 3 \mathrm{~K} 27 \mathrm{me} 3$ in the animal hemisphere whereas the genes preferentially expressed in the animal hemisphere do not have such marks in whole Xenopus embryos (Akkers et al., 2009). Constitutive expression of $x$ tjmjd3 and/or xtutx may be responsible for maintaining the demethylated state of the active genes in the animal hemisphere, and their continuous expression in the developing neuroectoderm in the early neurula is consistent with the requirement of Jmjd3 in the neural commitment of ES cells (Burgold et al., 2008). Expression of $x t j m j d 3$ was also shown to continue from the marginal zone to the notochord lineage, where a T-box gene for mesoderm formation, brachyury/T, is expressed (Fig. 3F) (Smith et al., 1991). As shown in ES cells, XtJMJD3 may be directly involved in the activation of the brachyury/tgene by interacting with NODAL-activated SMAD2/3 in these tissues (Dahle et al., 2010).

The details of our expression analysis suggest many unexplored roles for jmjd3, utx and ezh2 in the development of specific tissues, such as the retina, lens and inner ear. It is also noteworthy that their expression patterns are very similar throughout development, from early body axis formation to late oraganogenesis, even though demethylases and methylases are thought to antagonize each other. Thus, we speculate that xtjmjd3, xtutx and xtezh2 may be, in part, co-regulated to allow for their cooperation. The progressive changes of gene expression during development likely require the simultaneous action of both the demethylase and methylase; the former de-represses silent genes to activate new genetic programs while the latter silences active genes to shutdown old programs. This possibility will be explored in future analyses of the regulatory mechanisms controlling these epigenetic regulators and the mechanisms governing their recruitment to target gene loci.

\section{Materials and Methods}

\section{Plasmid construction}

The xtjmjd3 cDNA clone, BC167994, was obtained from Open Biosystems. The 1656 bp partial cDNA fragment of $x$ tjmjd3 was amplified by PCR from a cDNA pool of $X$. tropicalis tailbud embryos (stages 27-28) using the primers 5'-ATGTTCTAGAGGGGCAGAAGTATTGC-3' and 5'GCTTCTCTCGTGGAAGGTTCTG-3'. The 1420 bp Xbal-Sacl region from this cDNA fragment was introduced into BC167994 in place of the corresponding $X$ bal-Sacl region containing the adenine insertion to reconstruct the 5220 bp full-length coding region of $x t j m j d 3$ without the frame shift mutation. The resulting $x t j m j d 3$ cDNA was subcloned into an expression vector, pCS2+, to generate pCS2+xtjmjd3. pGEM-xtjmjd3 was generated by subcloning part of the xtjmjd3 cDNA into the pGEM-T Easy vector (Promega). The cDNA fragment used for this subcloning was amplified from pCS2+xtjmjd3 using the primers 5'-TGACCGGATCTTGGTGGCC-3' and 5'-TCACCGGATGTTCGGGGGT-3'. pCS2+mJmjd3 was constructed by introducing the full coding region of the mouse Jmjd3 cDNA that was excised from BC075632 (Open Biosystems) into pCS2+.

For the cloning of xtutx and xtezh2 cDNAs, we designed the following primer pairs according to the sequences of NM001130335 and NM001017293: xtutx, 5'-GGTCCGGGAGATTGTGAATGG-3' and 5'-TCAGGATGATGGTGAAGGCATT-3'; xtezh2, 5'-GGTTGATTGGTACCAAGACATGC-3' and 5'-TCAGGGGATTTCCATTTCTCTC-3'. Using these primer pairs, a 729 bp cDNA fragment of $x t u t x$ and an 881 bp cDNA fragment of $x$ tezh2 were amplified from the cDNA pool of $X$. tropicalis tailbud embryos and cloned into a pGEM-T Easy vector. The resulting plasmids, pGEM-xtutx and pGEM-xtezh2, were verified by sequencing. The expression vector containing the complete coding sequence for $x$ tutx, pCMVSPORT6-xtutx (BC167610), was obtained from Open Biosystems and verified by sequencing. 


\section{Cell culture and fluorescent immunostaining}

Transient transfection into COS-7 cells was performed using FuGene6 (Promega) with $1.5 \mu \mathrm{g}$ of pCS2+, pCS2+xtjmjd3, pCMVSPORT6-xtutx, or pCS2+mJmjd3, along with $0.5 \mu \mathrm{g}$ of a tracer plasmid, pCAGGS+GFP (Ogino and Yasuda, 1998), in a 35-mm culture dish. After a 36-hr incubation, the cells were fixed for fluorescent immunostaining as previously described (Hong et al., 2007). A 1/500 diluted rabbit anti-H3K27me3 antibody (Millipore, 07449) and a 1/1000 diluted mouse anti-GFP antibody (Santa Cruz Biotechnology, sc-9996) were used as primary antibodies in conjunction with 1/1000 diluted Alexa 488-conjugated goat anti-rabbit IgG (Invitrogen, A11001) and 1/1000 diluted Alexa 568-conjugated goat anti-mouse IgG (Invitrogen, A11011) secondary antibodies, respectively. After the antibody reactions, the cells were mounted with VECTASHIELD Mount Medium with DAPI (Vector). The images were acquired using a laser-scanning confocal microscope (Carl Zeiss, LSM710).

\section{$R T-P C R$ analysis and in situ hybridization}

The mRNAs were extracted from $X$. tropicalisembryos using the RNeasy kit (Qiagen). cDNA synthesis from these mRNAs and subsequent PCR reactions were performed using the PrimeScript 1st strand cDNA Synthesis kit (Takara) and AmpliTaq Gold 360 Master Mix (Applied Biosystems). Primer pairs and corresponding numbers of PCR cycles were as follows: xtjmjd3, 5'-GCTCCTCCAGCACTTGTACCTT-3' and 5'- TAGATCTGACCCAGCTGCTCC-3', 25 cycles; xtutx, 5'-GGTCCGGGAGATTGTGAATGG-3' and 5'TCAGGATGATGGTGAAGGCATT-3', 30 cycles; $x t e z h 2,5$ '- CCTGAAAATGTGGAATGGAG-3' and 5'-CCCCACAAGTTAAACACAGG-3', 30 cycles; $X$. tropicalis odc ( $x$ todc), 5'- GTGGAGCAAAAGATCAATGAAGTGTCC-3' and 5'-CAGCTAGCTGCGTATTTGATCTGG-3', 25 cycles. Each primer pair was designed to hybridize to exons separated by an intron(s) in order to distinguish whether the resulting amplicons were derived from the cDNA or contamination with genomic DNA.

The digoxigenin-labeled antisense probes for in situ hybridization analyses of $x t j m j d 3, x t u t x$ and $x t e z h 2$ were generated using pGEM-xtjmjd3, pGEM-xtutx and pGEM-xtezh2, respectively. The probe for bracyury was generated using pSP73-Xbra (Smith et al., 1991). In situ hybridization was performed as previously described (Sive et al., 2000). The blastula, gastrula and early neurula embryos were subjected to whole-mount in situ hybridization and then sectioned using a microslicer (DTK-1000, Dosaka EM Co.). The tailbud embryos were subjected to both whole-mount and section in situ hybridization, and tadpoles were subjected to section in situ hybridization.

\section{Acknowledgements}

We thank Mr. Makoto Okano for his early contributions to this work. We also thank Dr. Yoshiko Takahashi and members of the Takahashi lab for helpful comments on this manuscript. This work was supported by Grant-in-Aid for Scientific Research (C) (20579002 and 23570256) from the Japan Society for the Promotion of Science (JSPS), Grant-in-Aid for Scientific Research on Innovative Areas (21200064) from the Ministry of Education, Culture, Sports, Science and Technology (MEXT) of Japan, grants from the NAIST Foundation and Takeda Science Foundation to $\mathrm{H}$. Ogino, by Grant-in-Aid for Young Scientists (B) (21770234) from JSPS, Research for Promoting Technological Seeds (A) (10-099) from JST, and research grants from the Inamori foundation and the Sasakawa foundation to H. Ochi, and by CREST (JST), and the Global COE Program in NAIST (Frontier Biosciences: strategies for survival and adaptation in a changing global environment).

\section{References}

AKKERS, R.C., VAN HEERINGEN, S.J., JACOBI, U.G., JANSSEN-MEGENS, E.M., FRANCOIJS, K.J., STUNNENBERG, H.G. and VEENSTRA, G.J. (2009).
A hierarchy of $\mathrm{H} 3 \mathrm{~K} 4 \mathrm{me} 3$ and $\mathrm{H} 3 \mathrm{~K} 27 \mathrm{me} 3$ acquisition in spatial gene regulation in Xenopus embryos. Dev Cell 17: 425-434.

ALDIRI, I. and VETTER, M.L. (2009). Characterization of the expression pattern of the PRC2 core subunit Suz12 during embryonic development of Xenopus laevis. Dev Dyn 238: 3185-3192.

BARNETT, M.W., SEVILLE, R.A., NIJJAR, S., OLD, R.W. and JONES, E.A. (2001). Xenopus Enhancer of Zeste (XEZ); an anteriorly restricted polycomb gene with a role in neural patterning. Mech Dev 102: 157-167.

BEISEL, C. and PARO, R. (2011). Silencing chromatin: comparing modes and mechanisms. Nat Rev Genet 12: 123-135.

BERNSTEIN, B.E., MIKKELSEN, T.S., XIE, X., KAMAL, M., HUEBERT, D.J., CUFF, J., FRY, B., MEISSNER, A., WERNIG, M., PLATH, K. et al., (2006). A bivalent chromatin structure marks key developmental genes in embryonic stem cells. Cell 125: 315-326.

BURGOLD, T., SPREAFICO, F., DE SANTA, F., TOTARO, M.G., PROSPERINI, E., NATOLI, G. and TESTA, G. (2008). The histone H3 lysine 27-specific demethylase Jmjd3 is required for neural commitment. PLoS One 3: e3034.

DAHLE, O., KUMAR, A. and KUEHN, M.R. (2010). Nodal signaling recruits the histone demethylase Jmjd3 to counteract polycomb-mediated repression at target genes. Sci Signal 3: ra48.

DE SANTA, F., TOTARO, M.G., PROSPERINI, E., NOTARBARTOLO, S., TESTA, G and NATOLI, G. (2007). The histone H3 lysine-27 demethylase Jmjd3 links inflammation to inhibition of polycomb-mediated gene silencing. Cell 130: 1083-1094.

GREENFIELD, A., CARREL, L., PENNISI, D., PHILIPPE, C., QUADERI, N., SIGGERS, P., STEINER, K., TAM, P.P., MONACO, A.P., WILLARD, H.F. et al., (1998). The UTXgene escapes X inactivation in mice and humans. Hum Mol Genet7: 737-742.

HELLSTEN, U., HARLAND, R.M., GILCHRIST, M.J., HENDRIX, D., JURKA, J., KAPITONOV, V., OVCHARENKO, I., PUTNAM, N.H., SHU, S., TAHER, L. et al., (2010). The genome of the Western clawed frog Xenopus tropicalis. Science 328: $633-636$

HONG, S., CHO, Y.W., YU, L.R., YU, H., VEENSTRA, T.D. and GE, K. (2007) Identification of JmjC domain-containing UTX and JMJD3 as histone H3 lysine 27 demethylases. Proc Natl Acad Sci USA 104: 18439-18444.

HUBNER, M.R. and SPECTOR, D.L. (2011). Role of H3K27 Demethylases Jmjd3 and UTX in Transcriptional Regulation. Cold Spring Harb Symp Quant Biol.

LAIBLE, G., WOLF, A., DORN, R., REUTER, G., NISLOW, C., LEBERSORGER, A., POPKIN, D., PILLUS, L. and JENUWEIN, T. (1997). Mammalian homologues of the Polycomb-group gene Enhancer of zestemediate gene silencing in Drosophila heterochromatin and at S. cerevisiae telomeres. Embo J 16: 3219-3232.

LAN, F., BAYLISS, P.E., RINN, J.L., WHETSTINE, J.R., WANG, J.K., CHEN, S., IWASE S., ALPATOV, R., ISSAEVA, I., CANAANI, E. et al., (2007). A histone H3 lysine 27 demethylase regulates animal posterior development. Nature 449: 689-694.

MARGUERON, R. and REINBERG, D. (2011). The Polycomb complex PRC2 and its mark in life. Nature 469: 343-349.

NEWPORT, J. and KIRSCHNER, M. (1982). A major developmental transition in early Xenopus embryos: I. characterization and timing of cellular changes at the midblastula stage. Cell 30: 675-686.

OGINO, H. and YASUDA, K. (1998). Induction of lens differentiation by activation of a bZIP transcription factor, L-Maf. Science 280: 115-118.

SCHLOSSER, G. (2006). Induction and specification of cranial placodes. Dev Biol 294: 303-351.

SINDELKA, R., FERJENTSIK, Z. and JONAK, J. (2006). Developmental expression profiles of Xenopus laevis reference genes. Dev Dyn 235: 754-758.

SIVE, H., GRAINGER, R. and HARLAND, R. (2000). Early Development of Xenopus laevis-ALABORATORYMANUAL. Cold Spring Harbor Laboratory Press, New York.

SMITH, J.C., PRICE, B.M., GREEN, J.B., WEIGEL, D. and HERRMANN, B.G. (1991) Expression of a Xenopus homolog of Brachyury $(T)$ is an immediate-early response to mesoderm induction. Cell 67: 79-87.

SPEMANN, H. and MANGOLD, H. (1924). Über Induktion von Embryonalanlagen durch Implantation artfremder Organisatoren. Arch. Mikrosk. Anat. Entwicklungsmechan 100: 599-638. 


\section{Further Related Reading, published previously in the Int. J. Dev. Biol.}

Chromatin states of developmentally-regulated genes revealed by DNA and histone methylation patterns in zebrafish embryos Leif C. Lindeman, Cecilia L. Winata, Håvard Aanes, Sinnakaruppan Mathavan, Peter Aleström and Philippe Collas Int. J. Dev. Biol. (2010) 54: 803-813

Mechanisms of transcriptional repression by histone lysine methylation Philip Hublitz, Mareike Albert and Antoine H.F.M. Peters Int. J. Dev. Biol. (2009) 53: 335-354

Histone deacethylase 1 and 2-controlled embryonic development and cell differentiation Reinhard Brunmeir, Sabine Lagger and Christian Seiser Int. J. Dev. Biol. (2009) 53: 275-289

Interplay between DNA methylation, histone modification and chromatin remodeling in stem cells and during development

Kohta Ikegami, Jun Ohgane, Satoshi Tanaka, Shintaro Yagi, and Kunio Shiota Int. J. Dev. Biol. (2009) 53: 203-214

Role of polycomb proteins Ring1A and Ring1Bin the epigenetic regulation of gene expression

Miguel Vidal

Int. J. Dev. Biol. (2009) 53: 355-370

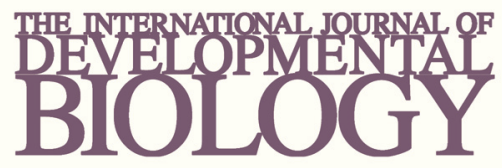

Volume 54 Nos. 6/7
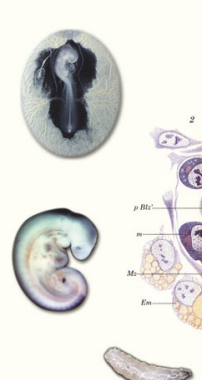

Developmental Hematopoiesis
Special Issue
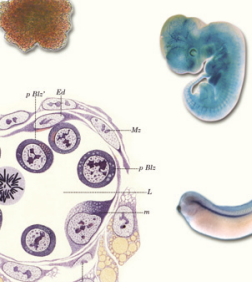

(1)

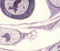

\section{.}

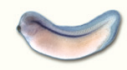

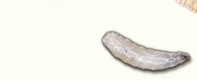

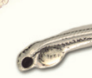

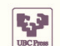

5 yr ISI Impact Factor $(2010)=2.961$

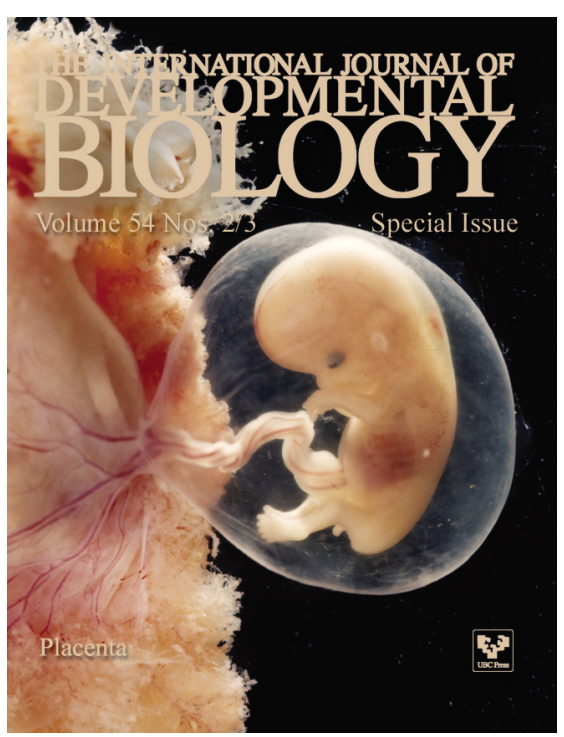

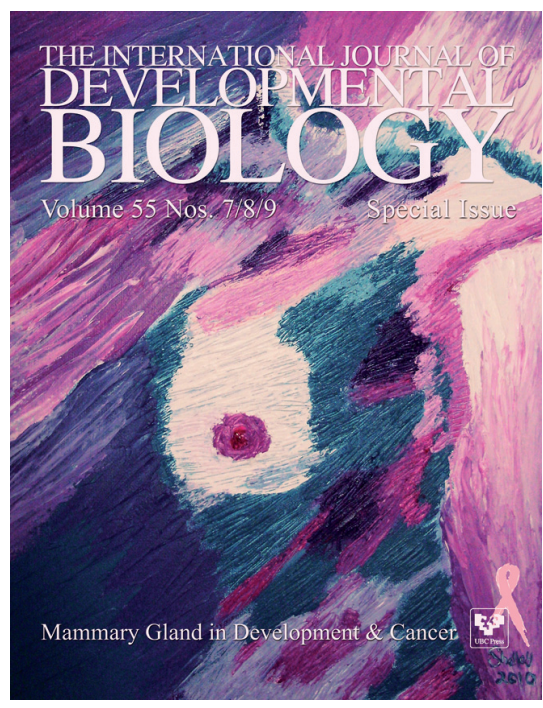

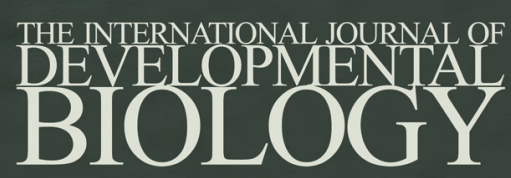

Volume 55 Nos. $4 / 5$

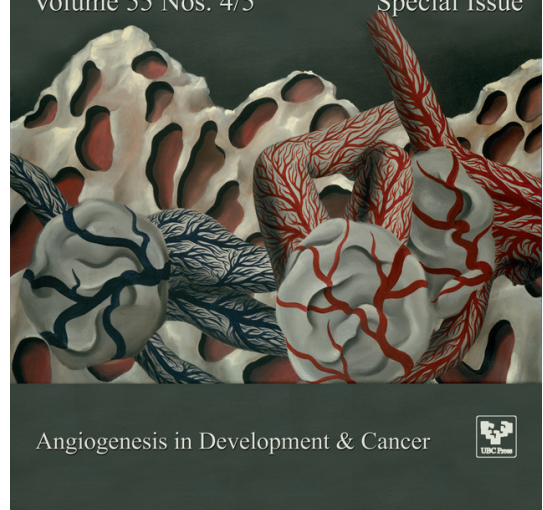

